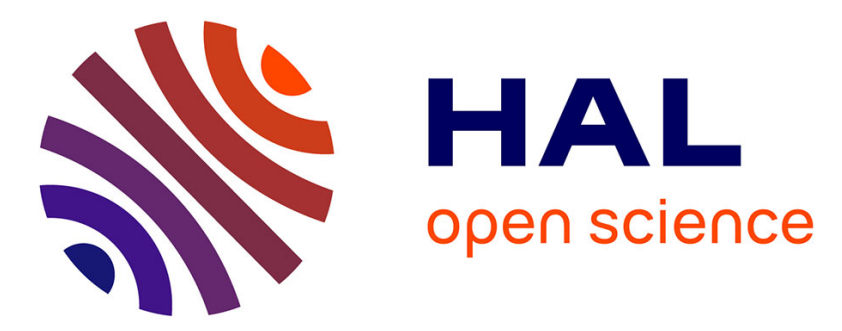

\title{
A comparison of performance of Deca Iron and Triple Deca Iron ultra-triathletes
}

\author{
Beat Knechtle, Thomas Rosemann, Romuald Lepers
}

\section{To cite this version:}

Beat Knechtle, Thomas Rosemann, Romuald Lepers. A comparison of performance of Deca Iron and Triple Deca Iron ultra-triathletes. 10.1097/01.CSMR.0000306201.49315.73 . inserm-01122252 SpringerPlus, 2014, 3, pp.461.

HAL Id: inserm-01122252 https://www.hal.inserm.fr/inserm-01122252

Submitted on 3 Mar 2015

HAL is a multi-disciplinary open access archive for the deposit and dissemination of scientific research documents, whether they are published or not. The documents may come from teaching and research institutions in France or abroad, or from public or private research centers.
L'archive ouverte pluridisciplinaire HAL, est destinée au dépôt et à la diffusion de documents scientifiques de niveau recherche, publiés ou non, émanant des établissements d'enseignement et de recherche français ou étrangers, des laboratoires publics ou privés. 


\title{
A comparison of performance of Deca Iron and Triple Deca Iron ultra-triathletes
}

\author{
Beat Knechtle ${ }^{1,2^{*}}$, Thomas Rosemann ${ }^{1}$, Romuald Lepers ${ }^{3}$ and Christoph Alexander Rüst ${ }^{1}$
}

\begin{abstract}
This study intended to compare the performance of ultra-triathletes competing in a Deca Iron ultra-triathlon (i.e. 10 times $3.8 \mathrm{~km}$ swimming, $180 \mathrm{~km}$ cycling, and $42.2 \mathrm{~km}$ running) with the performance of athletes competing in a Triple Deca Iron ultra-triathlon (i.e. 30 times $3.8 \mathrm{~km}$ swimming, $180 \mathrm{~km}$ cycling, and $42.2 \mathrm{~km}$ running). Split and overall race times of six male finishers in a Deca Iron ultra-triathlon and eight male finishers in a Triple Deca Iron ultra-triathlon were analysed using multiple $t$-tests, linear and non-linear regression analyses, and analysis of variance. Among the 19 starters (i.e. 17 men and two women) in the Deca Iron ultra-triathlon, six men (i.e. 35.3\% of all starters) finished the race. The mean swimming, cycling, running and overall race times of the six finishers across the ten days were 1:19 \pm 0:09 h:min, 6:36 0 0:19 h:min, 6:03 \pm 0:47 h:min and 14:44 \pm 1:17 h:min, respectively. The times of the split disciplines and overall race time increased linearly across the ten days. Total transition times did not change significantly across the days and were equals to $48 \pm 8$ min. Among the 22 starters (i.e. 20 men and two women) in the Triple Deca Iron ultra-triathlon, eight men (i.e. $36.4 \%$ of all starters) finished. The mean swimming, cycling, running and overall race times of the eight finishers across the 30 days were 1:11 \pm 0:07 h:min, 6:19 \pm 0:32 h:min, 5:34 \pm 1:15 h:min and 13:44 \pm 1:50 h:min, respectively. Split and overall race times showed no change across the 30 days. Total transition times showed no change across the days and were equal to $41 \pm 11 \mathrm{~min}$. To summarize, the daily performance decreased across the ten days for the Deca Iron ultra-triathletes (i.e. positive pacing) while it remained unchanged across the 30 days for the Triple Deca Iron ultra-triathletes (i.e. even pacing).
\end{abstract}

Keywords: Triathlon; Swimming; Cycling; Running; Ultra-endurance

\section{Background}

Ultra-endurance performance is defined as any endurance performance of six hours or longer in duration (Zaryski and Smith 2005). An Ironman triathlon covering $3.8 \mathrm{~km}$ swimming, $180 \mathrm{~km}$ cycling and $42.2 \mathrm{~km}$ running with the fastest winner times of $\sim 8 \mathrm{hrs}$ has to be considered as an ultra-endurance performance (Lepers 2008). Apart from the classical Ironman triathlon held as a single stage race, also longer ultra-triathlons such as multi-stage races with the completion of consecutive Ironman triathlons held for several days are known (Herbst et al. 2011; Knechtle et al. 2011a).

In a Deca Iron ultra-triathlon - where each day an Ironman triathlon has to be finished for ten consecutive days performance decreased with increasing duration of the

\footnotetext{
* Correspondence: beat.knechtle@hispeed.ch

${ }^{1}$ Institute of Primary Care, University of Zurich, Zurich, Switzerland

${ }^{2}$ Gesundheitszentrum St. Gallen, Vadianstrasse 26, 9001 St. Gallen, Switzerland Full list of author information is available at the end of the article
}

race (Herbst et al. 2011; Knechtle et al. 2012a) where the fastest Ironman was achieved on the first day (Herbst et al. 2011; Knechtle et al. 2008a) and the slowest on the last day (Herbst et al. 2011). A multi-stage ultra-triathlon such as a Deca Iron ultra-triathlon is a highly selective race and less than $50 \%$ of the starters are able to finish (Herbst et al. 2011; Knechtle et al. 2012a). The most important predictor variables for a successful finish in a Deca Iron ultra-triathlon were extensive previous experience since the number of finished Triple Iron ultra-triathlons and the personal best time in a Triple Iron ultra-triathlon were related to overall race time (Herbst et al. 2011).

Since the first edition of a Deca Iron ultra-triathlon in 2006 (Knechtle et al. 2008a), several races of this kind have been held mainly in Monterrey, Mexico (Herbst et al. 2011; Knechtle et al. 2012a). However, in autumn 2013, ultraendurance triathletes in Lonato des Garda, Italy, intended to go for new limits by organizing a Triple Deca Iron ultratriathlon, where the athletes had to finish each day an 
Ironman triathlon for 30 consecutive days. In the same race, a second group of athletes competed in a separate Deca Iron ultra-triathlon to finish ten Ironman triathlons within ten consecutive days.

Little is known about the pacing strategy in ultraendurance performance (Abbiss and Laursen 2008). Actual evidence suggests that during endurance and ultraendurance performance well-trained athletes tend to adopt a positive pacing strategy, whereby after peak speed is reached, the athlete progressively slows (Abbiss and Laursen 2008). The underlying mechanisms influencing the regulation of pace during exercise are currently unclear (Abbiss and Laursen 2008). It has been suggested, however, that self-selected exercise intensity is regulated within the brain based on a complex algorithm involving peripheral sensory feedback and the anticipated workload remaining (St Clair Gibson et al. 2006).

To date, to the best of our knowledge, the Triple Deca Iron ultra-triathlon represents the longest event ever finished by triathletes combining swimming, cycling and running. However, in summer 2013, an athlete completed in Laval, Québec, Canada, for the first time in history in a self-paced race the total distance of 33 Ironman triathlons within 33 consecutive days (Knechtle et al. 2014). The athlete finished the total distance of 7,458 km (i.e. $125 \mathrm{~km}$ swimming, 5,940 km cycling and 1,393 km running) within $410 \mathrm{~h}$ and finished each Ironman triathlon in a mean time of 12:27 h:min. During the 33 days, the athlete became slower in swimming, transition time 1 , and transition time 2 . However, in cycling, running and overall race time, the athlete was able to maintain his performance during the 33 days (Knechtle et al. 2014). The question is now whether athletes competing in an official race covering 30 Ironman triathlons within 30 days would be able to maintain their performance during one month as this athlete showed in 33 days in his self-paced race. The aim of the present study was therefore to compare the changes in performance over days for both Triple Deca Iron ultra-triathletes and Deca Iron ultra-triathletes. Based upon previous findings in field studies for Deca Iron ultra-triathletes and in the case study with the 33 Ironman triathlons it was hypothesized that performance would decrease in Deca Iron ultratriathletes, but not in Triple Deca Iron ultra-triathletes.

\section{Methods}

\section{Ethics}

This study was approved by the Institutional Review Board of St. Gallen, Switzerland, with a waiver of the requirement for informed consent given that the study involved the analysis of publicly available data.

\section{The races}

The Deca Iron ultra-triathlon race consisted in performing one Ironman distance triathlon (i.e. $3.8 \mathrm{~km}$ swimming,
$180 \mathrm{~km}$ cycling and $42 \mathrm{~km}$ running) daily for ten consecutive days whereas the Triple Deca Iron ultra-triathlon consisted in performing one Ironman distance triathlon daily for 30 consecutive days. The races were held in and around 'Parco La Quiete' (www.parcolaquiete.it) in Lonato del Garda in the North of Italy and south to Lake Garda. The Triple Deca Iron ultra-triathlon started on September 8th, 2013, and the Deca Iron ultra-triathlon started 20 days later on September 28th, 2013. Swimming was held in a non-heated 25-m out-door pool where wetsuits were allowed. Cycling was performed as a $180-\mathrm{km}$ non-drafting time trial on open roads in the hilly surroundings near 'Parco La Quiete' on laps of $6 \mathrm{~km}$. Running was held around the lake in 'Parco La Quiete' on flat laps of one $\mathrm{km}$ on grass $(\sim 50 \%)$ and stone slabs $(\sim 50 \%)$. Laps in swimming were counted manually by the staff of the race direction. Laps in cycling and running were counted electronically by using a chip system. On Day 7, cycling laps were counted manually due to problems with the electronic chip system. In the first 21 days, air temperature was at $\sim 25-30^{\circ} \mathrm{C}$ and water temperature at $\sim 25^{\circ} \mathrm{C}$. In the last nine days, weather conditions changed considerably where air temperature continuously dropped to $\sim 14^{\circ} \mathrm{C}$ and water temperature dropped to $\sim 17^{\circ} \mathrm{C}$ by the end of the race. In the last three days of the race, rain was continuously falling.

\section{Data collection and data analyses}

The data set for this study was obtained from the race director for electronically recorded split times. Overall race times and split times (i.e. $3.8 \mathrm{~km}$ swimming, $180 \mathrm{~km}$ cycling and $42 \mathrm{~km}$ running) of all female and male starters were analysed regarding changes over days. Each set of data was tested for normal distribution (D'Agostino and Pearson omnibus normality test) and for homogeneity of variances (Levene's test) before statistical analyses. Since the change in sex difference in endurance is assumed to be non-linear (Reinboud 2004), we calculated both the linear and the non-linear regression models that fit the data best. We compared the best-fit non-linear models to the linear models using Akaike's Information Criteria (AIC) as well as F-test in order to show whether the linear or the non-linear model would be the most appropriate to explain the trend of the data. Differences in absolute and relative performance between finishers in the Deca Iron ultra-triathlon and in the Triple Deca Iron ultra-triathlon were compared using multiple $t$-test analyses with individual analysis of each pair of Deca and Triple Deca Iron ultra-triathletes and with Holm-Sidak correction for multiple comparisons. Absolute and relative performance of the Deca Iron ultra-triathletes were compared with the performance of Day 1-10, Day 11-20 and Day 21-30 of the Triple Deca Iron ultra-triathletes using repeated measures one-way analysis of variance (ANOVA) with the 
Greenhouse-Geisser correction and Tukey's multiple comparison test with individual variances computed for each comparison. Statistical analyses were performed using CurveExpert Professional (Version 2.0.3, Hyams D.G.) and GraphPad Prism (Version 6.01, GraphPad Software, La Jolla, CA, USA). Significance was accepted at $p<0.05$ (two-sided for $t$-tests). Data in the text and figures are given as mean \pm standard deviation (SD).

\section{Results}

\section{Deca iron ultra-triathlon}

Among the 19 starters (i.e. 17 men and two women), six men (i.e. $35.3 \%$ of all starters) finished the race. Among the non-finishers, six triathletes stopped before the 6th day and seven triathletes stopped between the 6th and the 9th day. The mean swimming, cycling, running and overall race times of the six finishers across the ten days were 1:18 \pm 0:08 h:min, 6:35 \pm 0:37 h:min, 6:02 \pm 1:00 h: min and 14:44 \pm 1:44 h:min, respectively. All split times and overall race times increased linearly across the ten days (Figure 1). Total transition times did not change significantly across the days and were equals to $48 \pm$ $8 \mathrm{~min}$. The overall race time of the winner (i.e. the triathlete who had the fastest overall time after the ten days) was 129:33 h:min. His mean swimming, cycling, running and overall race times across the ten days were 1:06 0:01 h:min, 6:14 \pm 0:25 h:min, 5:06 $\pm 0: 16$ h:min and 12:57 \pm 0:35 h:min, respectively.

\section{Triple Deca iron ultra-triathlon}

Among the 22 starters (i.e. 20 men and two women), eight men (i.e. $36.4 \%$ of all starters) finished the 30
Ironman distance triathlon. Among the non-finishers, six triathletes finished between one and ten Ironman distances, five triathletes finished between 11 and 20 Ironman distances and three triathletes finished between 21 and 29 Ironman distances. The mean swimming, cycling, running and overall race times of the eight successful finishers across the 30 days were 1:10 \pm 0:07 h:min, 6:18 \pm 0:42 h:min, 5:33 \pm 1:17 h:min and 13:46 \pm 1:57 h: min, respectively. In contrast to the Deca Iron ultratriathlon, the times of the different disciplines and the total time did not change across the 30 days (Figure 2). The changes were non-linearly in swimming (i.e. polynomial regression 10th degree), cycling (i.e. polynomial regression 5th degree), running (i.e. polynomial regression 10th degree), and overall race time (i.e. polynomial regression 5th degree). Total transition times did not change significantly across the days and were equal to $41 \pm 11 \mathrm{~min}$. The overall race time of the winner (i.e. the triathlete who had the fastest overall race time after the 30 events) was 356:33 h:min. His mean swimming, cycling, running and overall race times across the 30 days were 1:12 \pm 0:03 h:min, 6:12 \pm 0:29 h:min, 3:56 $\pm 0: 20 \mathrm{~h}$ : min and 11:53 \pm 0:46 h:min, respectively.

\section{Comparison between the 10 days in the Deca with the $3 \times 10$ days in the Triple Deca}

Figure 3 presents the comparison of swimming, cycling, running, and overall race times for the Deca Iron ultratriathletes with the first ten days of the Triple Deca Iron ultra-triathletes expressed in absolute race times. There were no differences in swimming (Figure 3A) and running (Figure $3 \mathrm{C}$ ), but Triple Deca Iron ultra-triathletes
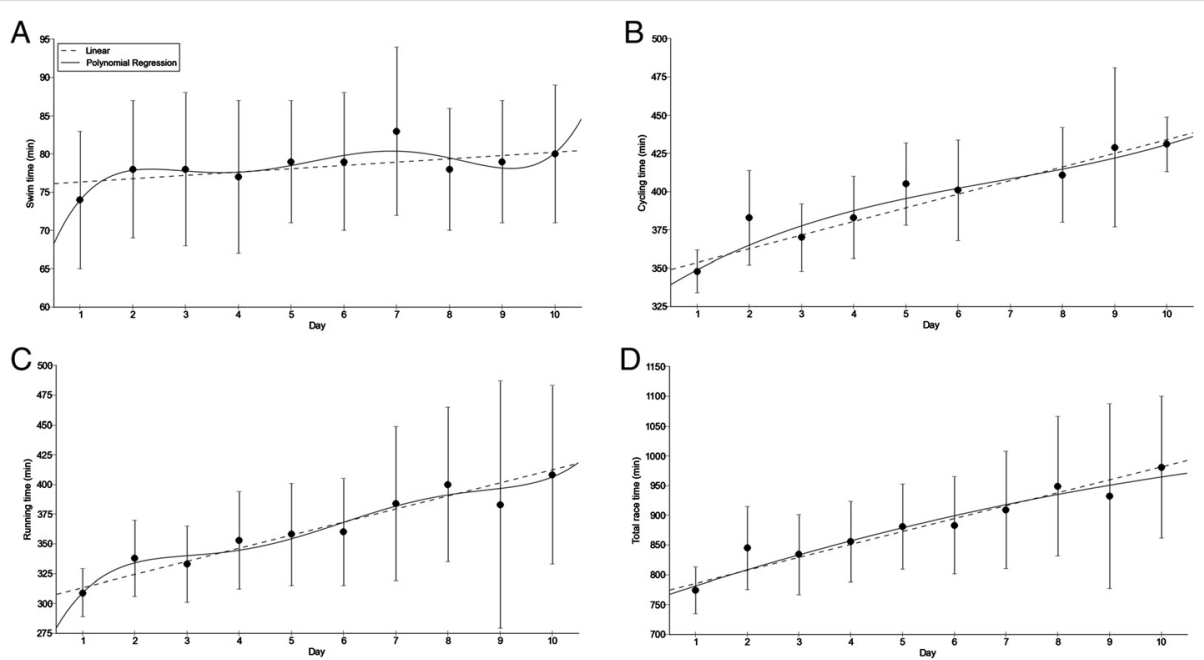

Figure 1 Changes in split and overall race times during the Deca Iron ultra-triathlon. (Panel A) swimming, (Panel B) cycling, (Panel C) running, (Panel D) overall race times $(n=6)$. Some cycling times on day 7 were not available. Values are means \pm SD. 

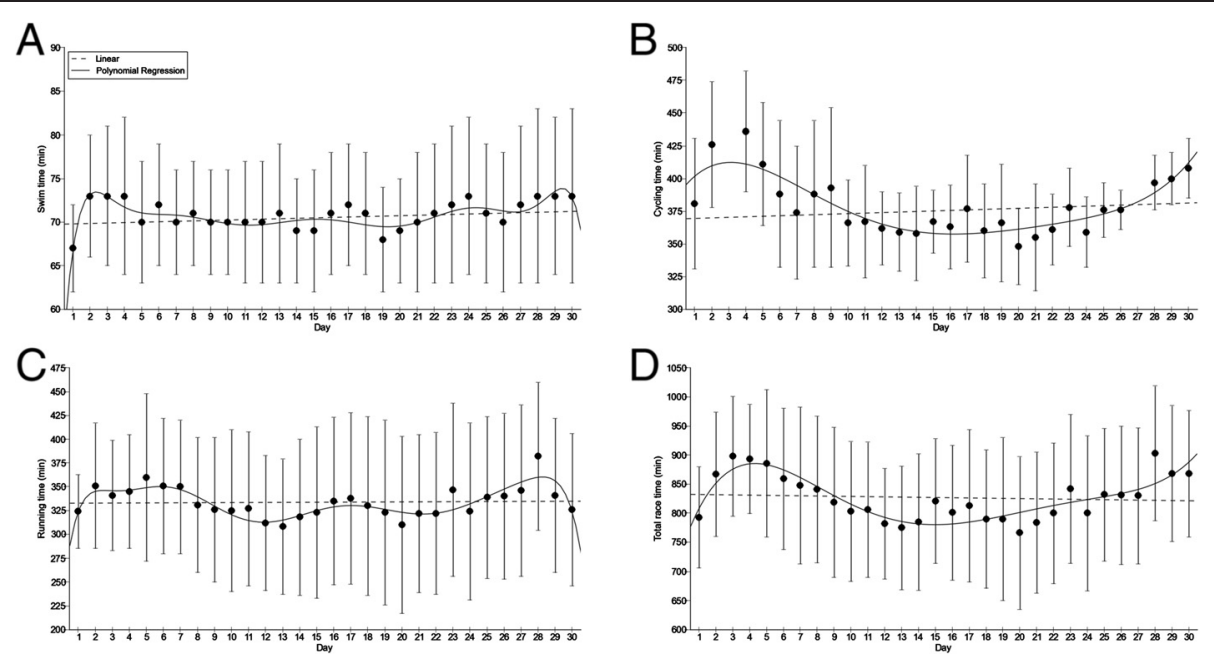

Figure $\mathbf{2}$ Changes in split and overall race times during the Triple Deca Iron ultra-triathlon. (Panel A) swimming, (Panel B) cycling, (Panel C) running, (Panel D) overall race times $(n=8)$. Some cycling times on day 3 and 27 were not available. Values are means \pm SD.

were faster in cycling (Figure 3B) on Day 10 than Deca Iron ultra-triathletes. Figure 4 presents the same comparisons for Day 11-20 in the Triple Deca Iron ultratriathlon with Day 1-10 in the Deca Iron ultra-triathlon. Similarly, the Triple Deca Iron ultra-triathletes were faster in cycling (Figure 4B) on Day 10 than the Deca Iron ultra-triathletes. Figure 5 compares Day 21-30 in the Triple Deca Iron ultra-triathlon with Day 1-10 in the Deca Iron ultra-triathlon. There were no differences in the performance between the finishers.

Figures 6, 7 and 8 show the comparisons in swimming, cycling and running expressed in percent of overall race
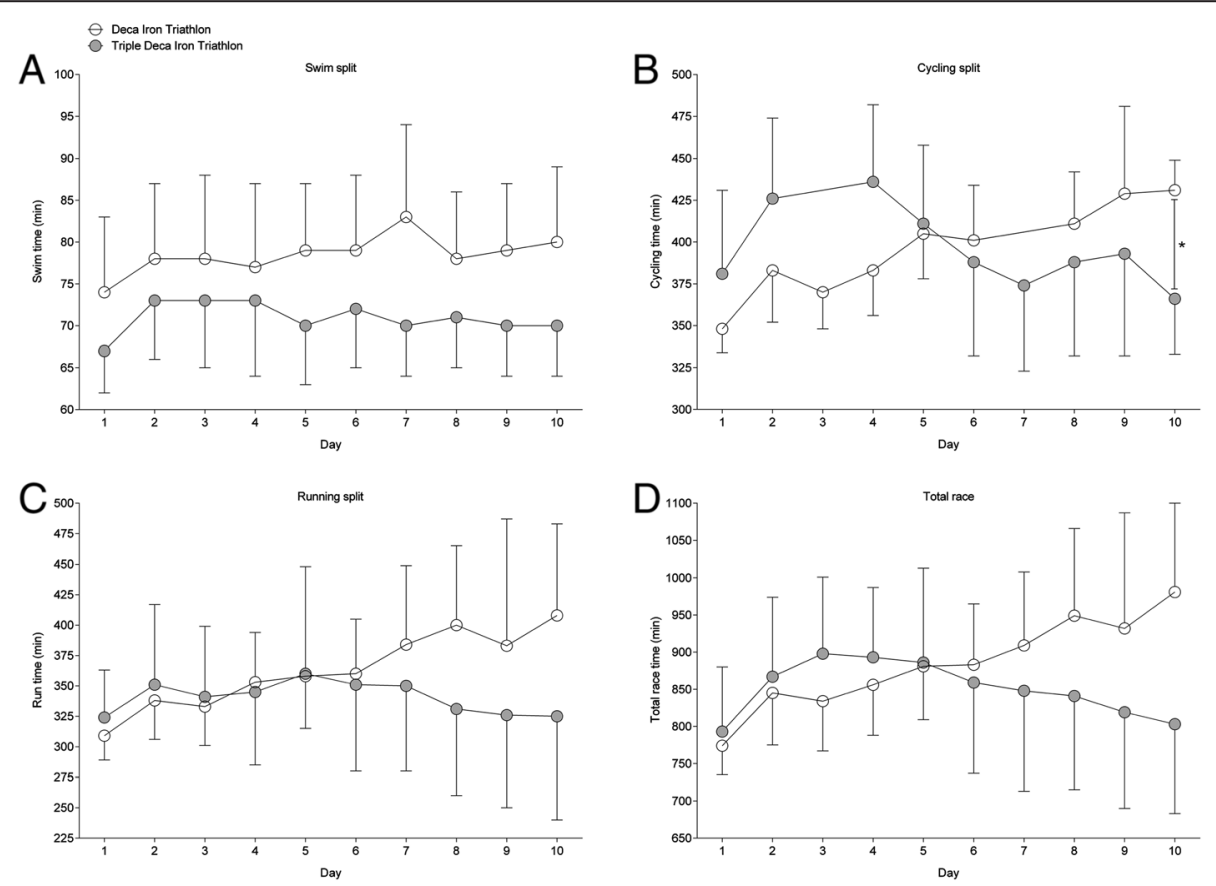

Figure 3 Comparison of split and overall race times for Deca Iron ultra-triathletes (i.e. all ten race days) and Triple Deca Iron ultra-triathletes (i.e. Day 1-Day 10) expressed in absolute race times. (Panel A) swimming, (Panel B) cycling, (Panel C) running, (Panel D) overall race times. Values are means $\pm S D$. 

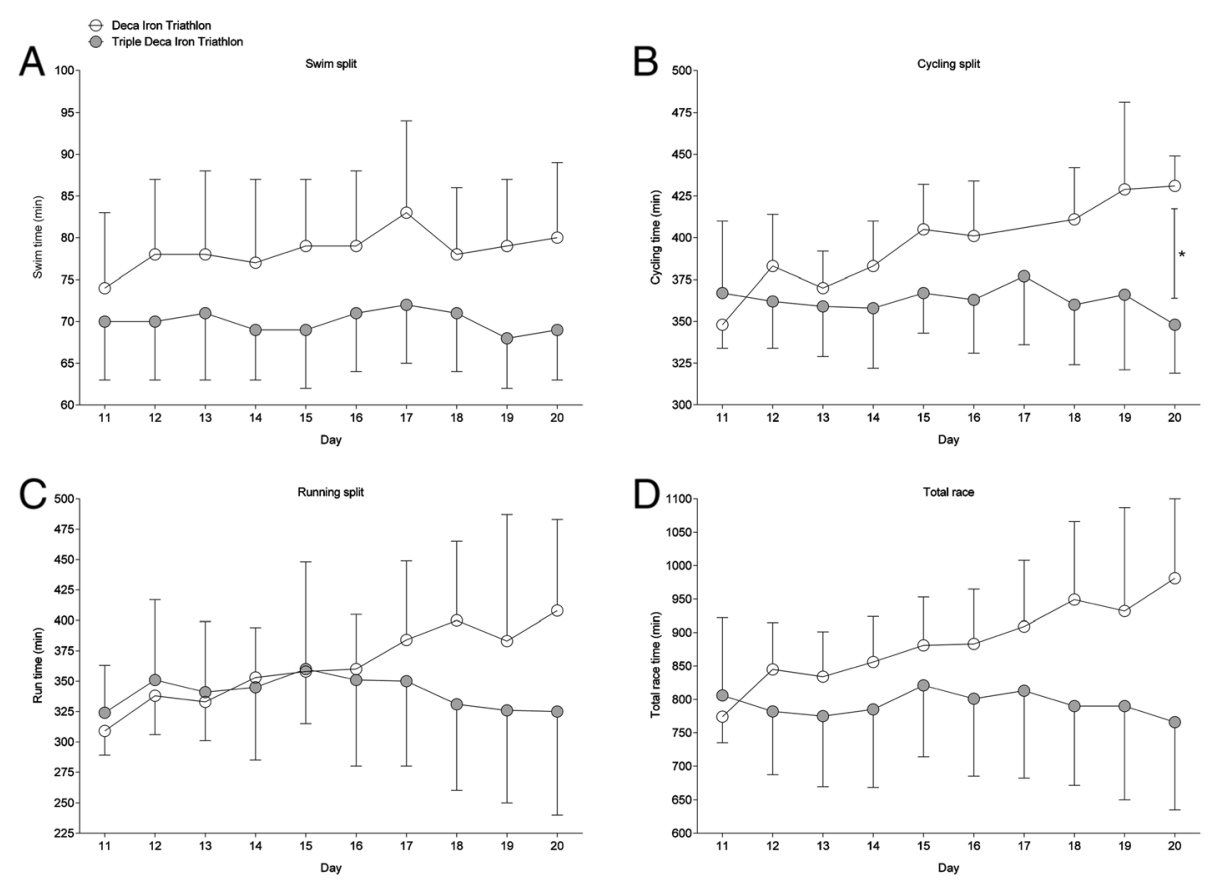

Figure 4 Comparison of split and overall race times for Deca Iron ultra-triathletes (i.e. all ten race days) and Triple Deca Iron ultra-triathletes (i.e. Day 11-Day 20) expressed in absolute race times. (Panel A) swimming, (Panel B) cycling, (Panel C) running, (Panel D) overall race times. Values are means \pm SD
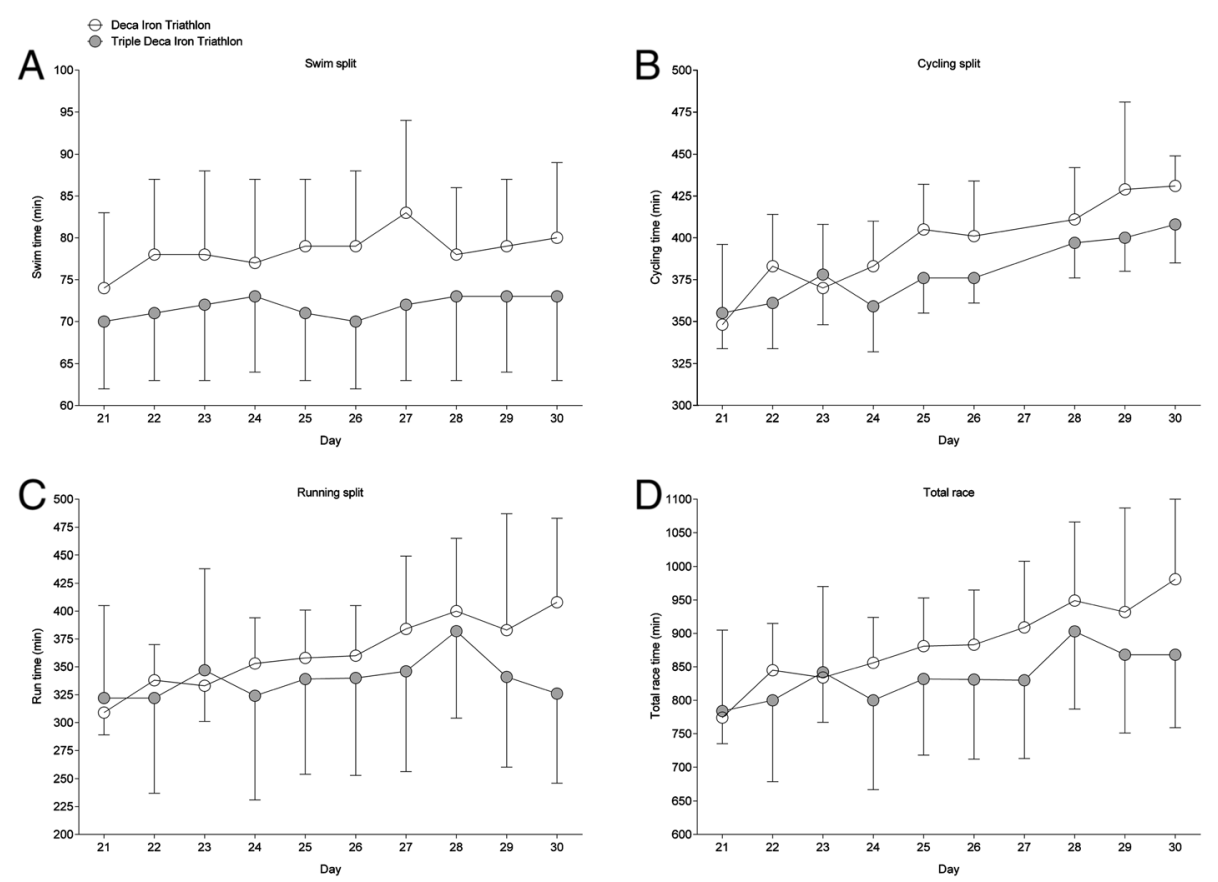

Figure 5 Comparison of split and overall race times for Deca Iron ultra-triathletes (i.e. all ten race days) and Triple Deca Iron ultra-triathletes (i.e. Day 21-Day 30) expressed in absolute race times. (Panel A) swimming, (Panel B) cycling, (Panel C) running, (Panel D) overall race times. Values are means $\pm \mathrm{SD}$. 

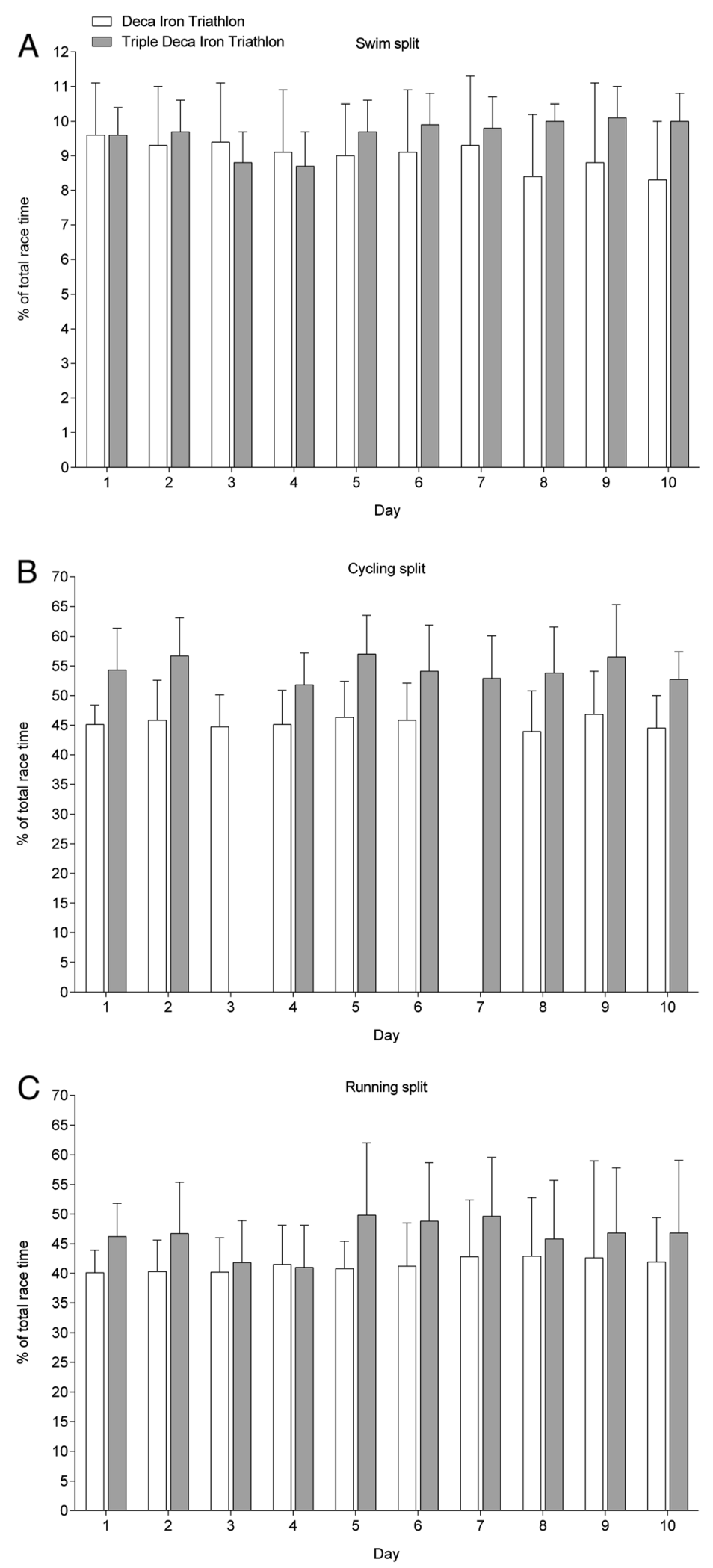

Figure 6 Comparison of split and overall race times for Deca Iron ultra-triathletes (i.e. all ten race days) and Triple Deca Iron ultra-triathletes (i.e. Day 1-Day 10) expressed in percent of overall race time. (Panel A) swimming, (Panel B) cycling, (Panel C) running. Values are means \pm SD. 

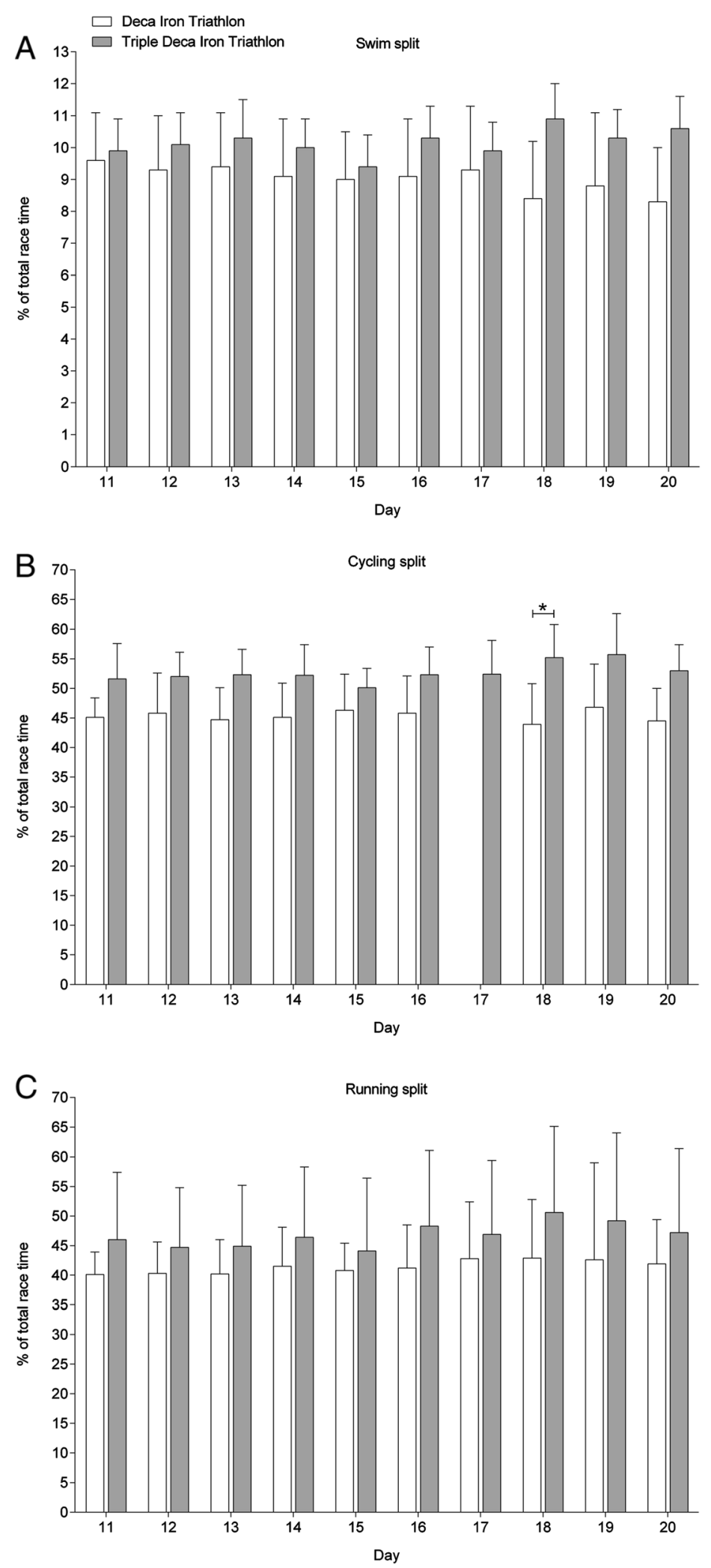

Figure 7 Comparison of split and overall race times for Deca Iron ultra-triathletes (i.e. all ten race days) and Triple Deca Iron ultra-triathletes (i.e. Day 11-Day 20) expressed in percent of overall race time. (Panel A) swimming, (Panel B) cycling, (Panel C) running. Values are means \pm SD. 

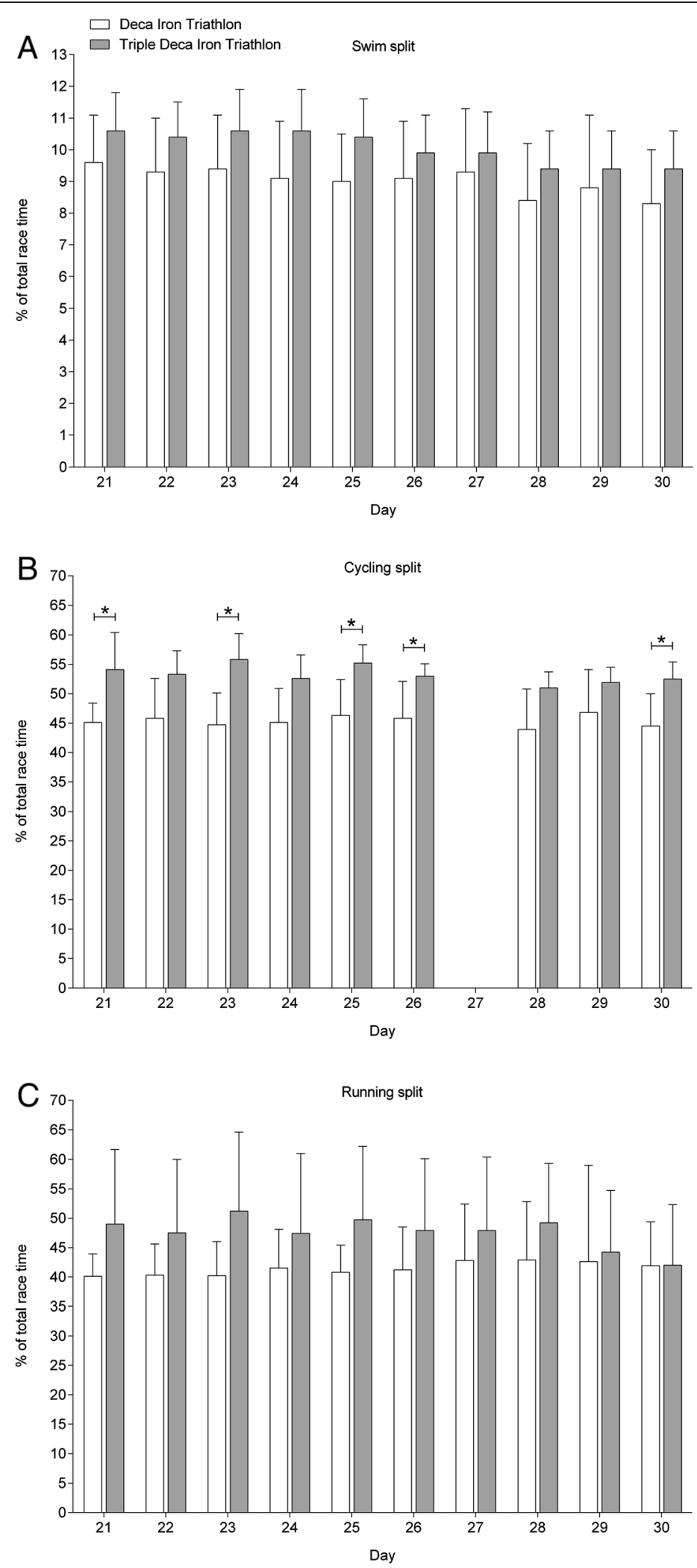

Figure 8 Comparison of split and overall race times for Deca Iron ultra-triathletes (i.e. all ten race days) and Triple Deca Iron ultra-triathletes (i.e. Day 21-Day 30) expressed in percent of overall race time. (Panel A) swimming, (Panel B) cycling, (Panel C) running. Values are means \pm SD. 
time for Day 1-10 in the Deca Iron ultra-triathlon with Day 1-10 (Figure 6), Day 11-20 (Figure 7) and Day 2130 (Figure 8) in the Triple Deca Iron ultra-triathlon. In the first ten days in the Triple Deca Iron ultra-triathlon, no differences were found compared to the ten days in the Deca Iron ultra-triathlon (Figure 6). When the second ten days in the Triple Deca Iron ultra-triathlon were compared to the ten days in the Deca Iron ultratriathlon, the Deca Iron ultra-triathletes were relatively faster on Day 18 in cycling (Figure 7). On Day 21-30, the athletes in the Deca Iron ultra-triathlon were relatively faster in cycling on Day 1, Day 3, Day 5, Day 6 and Day 10 compared to the Triple Deca Iron ultratriathletes (Figure 8).

Figure 9 presents the comparison in absolute times for swimming, cycling, running and overall race times of the ten days in the Deca Iron ultra-triathlon with Day 1-10, Day 11-20 and Day 21-30 in the Triple Deca Iron ultratriathlon. The athletes in the Deca Iron ultra-triathlon were relatively slower in swimming compared to Day 1-10 $(p<0.001)$, Day $11-20(p<0.001)$ and Day $21-30(p<0.001)$ in the Triple Deca Iron ultra-triathlon. In cycling, Triple Deca Iron ultra-triathletes were relatively faster than Deca Iron ultra-triathletes considering Day 11-20 $(p<0.05)$ and Day $21-30 \quad(p<0.05)$. The Triple Deca Iron ultratriathletes were relatively faster on Day 11-20 compared to Day $1-10(p<0.05)$. Considering running, Triple Deca Iron ultra-triathletes were relatively faster compared to Deca Iron ultra-triathletes on Day $11-20(p<0.05)$. And Triple Deca Iron ultra-triathletes were relatively faster on Day $11-20$ compared to Day $1-10(p<0.05)$. For overall race time, Triple Deca Iron ultra-triathletes were relatively faster on Day 1-10 $(p<0.01)$ and Day $11-20(p<0.05)$ compared to Deca Iron ultra-triathletes. Again, Triple Deca Iron ultra-triathletes were relatively faster on Day $11-20$ compared to Day $1-10(p<0.01)$.

Figure 10 presents the comparison for swimming, cycling, and running in percent of overall race time of the ten days in the Deca Iron ultra-triathlon with Day 1-10, Day 11-20, and Day 21-30 in the Triple Deca Iron ultratriathlon. Deca Iron ultra-triathletes were relatively faster in swimming compared to Triple Deca Iron ultra-triathletes on Day $11-20(p<0.01)$ and Day $21-30(p<0.001)$. In cycling, the Deca Iron ultra-triathletes were relatively faster compared to the Triple Deca Iron ultra-triathletes on Day $1-10(p<0.001)$, Day $11-20(p<0.001)$ and Day $21-30$ $(p<0.001)$. Considering running, the Deca Iron ultratriathletes were relatively faster on Day $1-10(p<0.01)$, Day $11-20(p<0.001)$ and Day 21-30 $(p<0.01)$ compared to the Triple Deca Iron ultra-triathletes.

\section{Discussion}

This study intended to analyse the changes in performance over time for Triple Deca Iron ultra-triathletes and Deca Iron ultra-triathletes and it was hypothesized that performance would decrease in Deca Iron ultratriathletes, but not in Triple Deca Iron ultra-triathletes. The most important finding was that the hypothesis was confirmed since performance decreased in Deca Iron ultra-triathletes but remained unchanged across time in Triple Deca Iron ultra-triathletes. The continuous decrease in performance in the Deca Iron ultra-triathletes
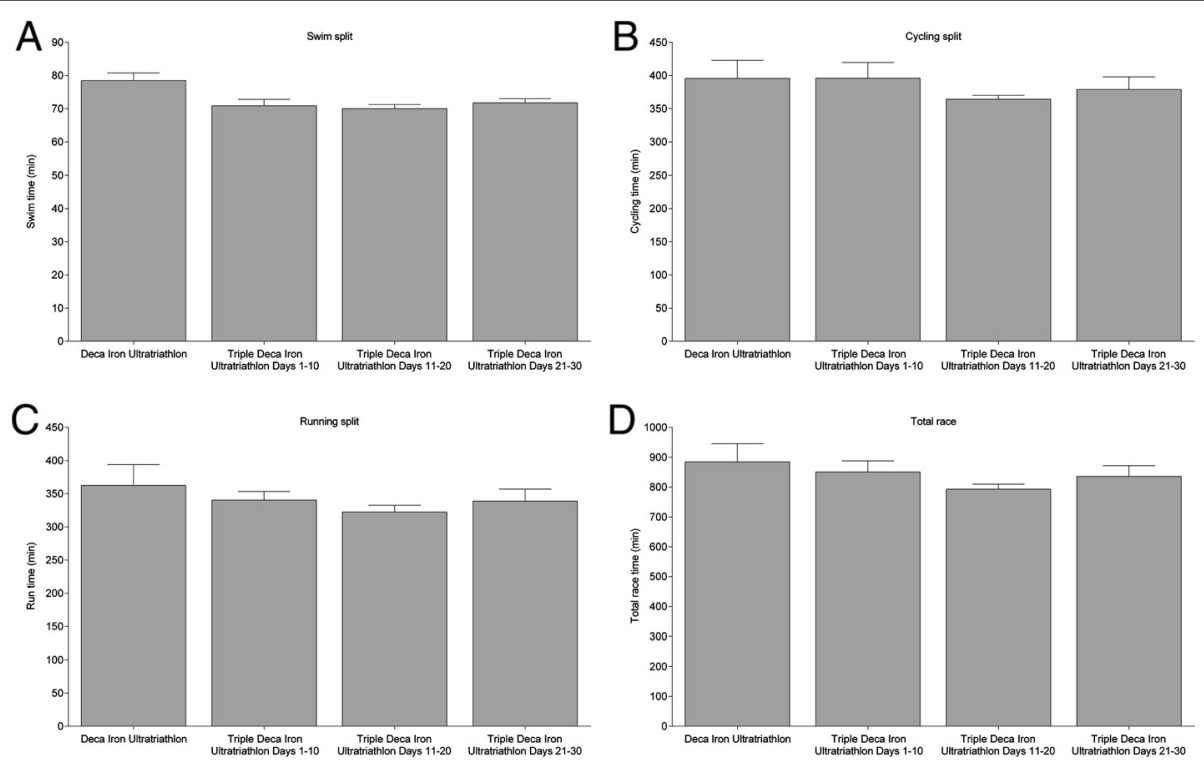

Figure 9 Comparison of split and overall race times for Deca Iron ultra-triathletes with Day 1-10, Day 11-20 and Day 21-30 in Triple Deca Iron ultra-triathletes in absolute race times. (Panel A) swimming, (Panel B) cycling, (Panel C) running, (Panel D) overall race times. Values are means \pm SD. 

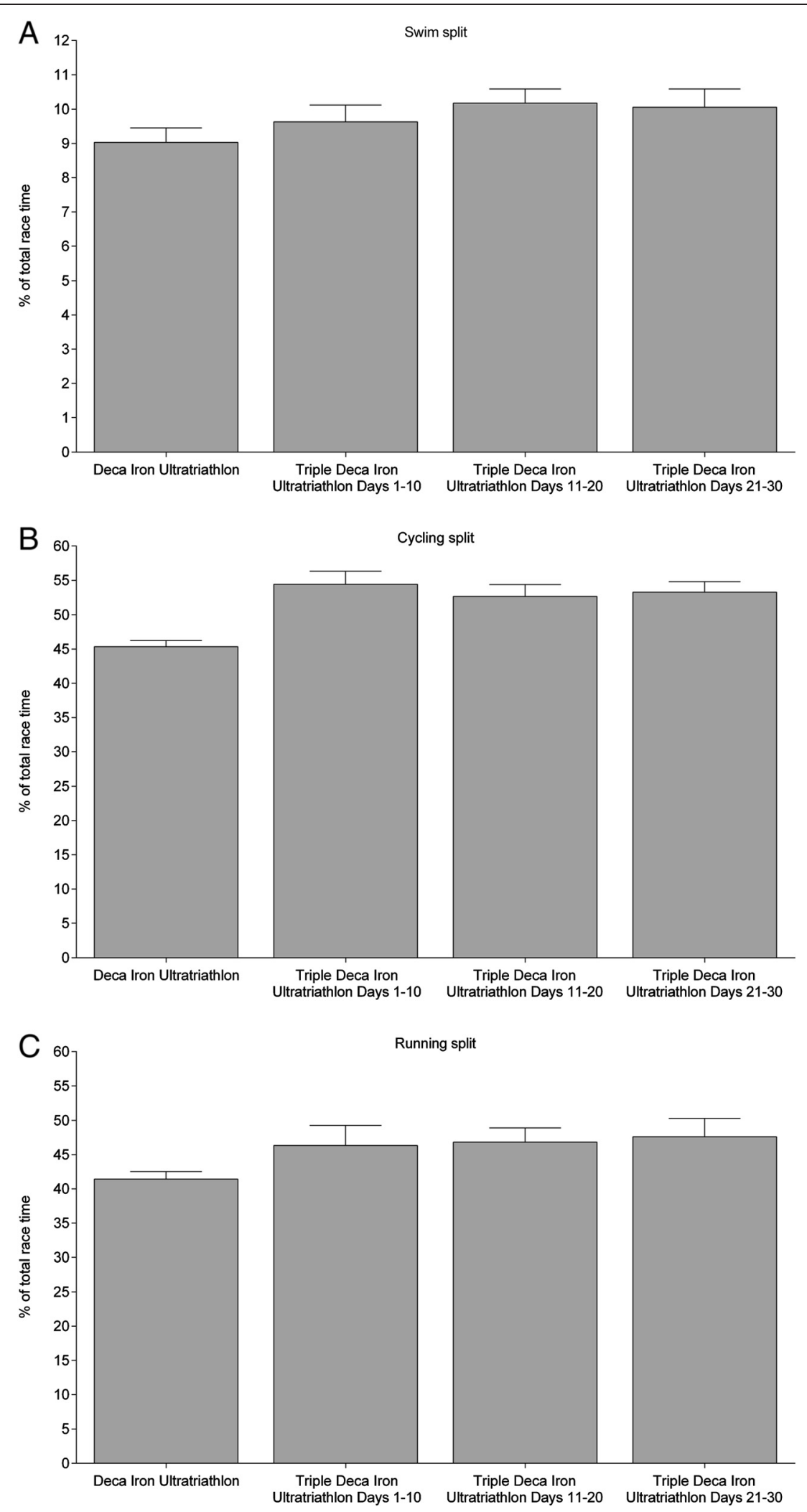

Figure 10 Comparison of split and overall race times for Deca Iron ultra-triathletes with Day 1-10, Day 11-20 and Day 21-30 in Triple Deca Iron ultra-triathletes in percent of overall race time. (Panel A) swimming, (Panel B) cycling, (Panel C) running. Values are means \pm SD. 
confirms previous findings observed for Deca Iron ultratriathletes competing in the 2006, 2007 and 2009 'World Challenge Deca Iron Triathlon' held in Monterrey, Mexico (Herbst et al. 2011). The unchanged performance in swimming, cycling, running and overall race time in the Triple Deca Iron ultra-triathletes confirms very recent findings in an athlete completing 33 Ironman triathlons within 33 days where the athlete was able to maintain his performance in cycling, running and overall race time during the 33 days (Knechtle et al. 2014).

\section{The aspect of pacing strategy}

An interesting observation was the difference in the comparison between absolute and relative performance. When the absolute daily performances of the Deca Iron ultra-triathletes were compared with the performances on Day 1-10, Day 11-20 and Day 21-30 of the Triple Iron ultra-triathletes, only minor differences were found. However, when the split performances were expressed relatively in percent of overall race times, Deca Iron ultra-triathletes were relatively faster in cycling in five of the ten days compared to the Triple Deca Iron ultra-triathletes in their last ten days. When the mean absolute performances of the ten days in the Deca Iron ultra-triathletes were compared to the performances on Day 1-10, Day 11-20 and Day 21-30 of the Triple Iron ultra-triathletes, the Deca Iron ultra-triathletes were slower in swimming, cycling and running compared to the specific segments of the Triple Deca Iron ultra-triathlon race. However, when the performances in the split disciplines was expressed in percent of overall race time, the Deca Iron ultra-triathletes were relatively faster in swimming, cycling and running compared to the three segments of the Triple Deca Iron ultra-triathlon race. A further interesting finding regarding absolute performances was that the Triple Iron ultra-triathletes were faster in the second segment of their race (i.e. Day 11-20) compared to their first segment (i.e. Day 1-10) in cycling, running, and overall performance. Generally, during ultraendurance events (i.e. endurance performances lasting for longer than six hours), athletes tend to adopt a positive pacing strategy (i.e. the athlete's speed gradually declines throughout the duration of the event) (Abbiss and Laursen 2008). The Triple Deca Iron ultra-triathletes, however, were able for a negative pacing in the first 20 days of the race. A potential explanation for the negative pacing (i.e. better performance in cycling, running and overall race times) could be the environmental conditions (i.e. unchanged course and habituation to the course), the habituation to the daily task for both athletes and support crews, and the stable weather conditions (i.e. no rain, constant air temperature of $\sim 25-30^{\circ} \mathrm{C}$ and constant water temperature of $2 \sim 5^{\circ} \mathrm{C}$ in the first 21 days). Mean absolute performances in the second (i.e. Day 11-20) and third segment (i.e. Day 21-30) were not different. Most probably the athletes were then very experienced and negated the deterioration of the environmental conditions (i.e. decrease in air and water temperature).

Overall, the Triple Deca Iron ultra-triathletes showed an 'even pacing' during the 30 days in contrast to the Deca Iron ultra-triathletes with a 'positive pacing' (Abbiss and Laursen 2008) during their ten days. Most probably the successful Triple Deca Iron ultra-triathletes choose the right constant pace in cycling in the first days to be able to successfully finish the whole race. And they were even able to improve cycling and running speed in the second segment of the race. The athletes in the Triple Deca Iron ultra-triathlon might have become accustomed during the first days to the burden of a daily Ironman. They may have learned to adopt a pacing strategy to finish the race successfully. A potential explanation could be that the successful finishers in the Triple Deca Iron ultra-triathlon went slower in the cycling part in order to save energy for the running split which could be due to higher pre-race experience in Triple Deca Iron ultra-triathletes compared to Deca Iron ultra-triathletes. Alternatively, the Deca Iron ultra-triathletes went too fast in the first days in cycling leading to different problems such as muscular problems, loss in stored energy in the muscles, muscle soreness forcing them to go slower in the following days.

\section{The aspect of previous experience}

An explanation for the differences in pacing strategy between the Deca Iron and the Triple Deca Iron ultratriathletes could be the previous experience of the successful finishers. Previous experience is an important predictor variable in an ultra-triathlon (Herbst et al. 2011; Lepers et al. 2011). It has been shown that race time in a Triple Iron ultra-triathlon was highly predictive for race time in a Deca Iron ultra-triathlon (Herbst et al. 2011; Lepers et al. 2011). Overall race time in a Deca Iron ultra-triathlon might be predicted by the equation Deca Iron ultra-triathlon race time $(\mathrm{min})=5885+3.69 \times$ race time in Triple Iron ultra-triathlon (minutes) (Lepers et al. 2011).

Since both the number and the personal best time in a Triple Iron ultra-triathlon were highly predictive for the performance in a Deca Iron ultra-triathlon (Herbst et al. 2011), we summarized for each finisher the number of completed Triple Iron ultra-triathlons with the personal best time with data available from the website of the International Ultra-Triathlon Association (IUTA). In addition to the data of Triple Iron ultra-triathlon, we inserted also the data from Double Iron ultra-triathlon and longer ultra-triathlons for both finishers in the Deca Iron ultra-triathlon (Table 1) and in the Triple Deca Iron ultra-triathlon (Table 2). In accordance with previous 
Table 1 Number of finished ultra-triathlons with personal best time for Double Iron, Triple Iron and longer distances for finishers in the Deca Iron ultra-triathlon

\begin{tabular}{lccccc}
\hline Rank & $\begin{array}{c}\text { Number of finished } \\
\text { Triple Iron } \\
\text { ultra-triathlons }\end{array}$ & $\begin{array}{c}\text { Personal best time } \\
\text { in Triple Iron } \\
\text { ultra-triathlon }\end{array}$ & $\begin{array}{c}\text { Number of finished } \\
\text { Double Iron } \\
\text { ultra-triathlons }\end{array}$ & $\begin{array}{c}\text { Personal best time } \\
\text { in Double Iron } \\
\text { ultra-triathlon }\end{array}$ & $\begin{array}{c}\text { Other completed } \\
\text { ultra-triathlons }\end{array}$ \\
\hline $\mathbf{1}$ & 24 & $37: 18 \mathrm{~h}: \mathrm{min}$ & 22 & $22: 57 \mathrm{~h}: \mathrm{min}$ & 3 Deca Iron with best time 240:55 h:min \\
$\mathbf{2}$ & - & - & 2 & $25: 10 \mathrm{~h}: \mathrm{min}$ & - \\
$\mathbf{3}$ & 1 & $47: 19 \mathrm{~h}: \mathrm{min}$ & 4 & $26: 34 \mathrm{~h}: \mathrm{min}$ & - \\
$\mathbf{4}$ & 1 & $55: 29 \mathrm{~h}: \mathrm{min}$ & - & - & - \\
$\mathbf{5}$ & - & - & - & - & - \\
$\mathbf{6}$ & 1 & $46: 45 \mathrm{~h}: \mathrm{min}$ & - & - & - \\
\hline
\end{tabular}

reports (Herbst et al. 2011; Knechtle et al. 2011b; Lepers et al. 2011) the winner in the Deca Iron ultra-triathlon had the highest number of finishes in both Triple and Double Iron ultra-triathlon and the fastest personal best times in both Triple and Double Iron ultra-triathlon (Table 1) compared to the other finishers. For the fastest finishers in the Triple Deca Iron ultra-triathlon, however, both the numbers and the personal best times in both Triple and Double Iron ultra-triathlon seemed not of relevance (Table 2). The fastest three finishers had focussed more in their previous races on longer ultratriathlon distances such as Deca Iron and Double Deca Iron ultra-triathlon. Similarly to the athletes in the Triple Deca Iron ultra-triathlon, the athlete completing 33 Ironman triathlons in 33 days in his self-paced race showed a broad experience in ultra-triathlon (Knechtle et al. 2014). He had finished 11 Double Iron ultratriathlons with a personal best time of 21:48 h:min, six Triple Iron ultra-triathlons with a personal best time of 36:29 h:min and one Deca Iron ultra-triathlon within 297:42 h:min (Knechtle et al. 2014).

\section{Limitations}

This study analysed the changes in performance in split and overall race times in Deca Iron and Triple Deca Iron ultra-triathletes. Unfortunately, aspects such as nutrition (Dempster et al. 2013; Knechtle et al. 2008b), fluid metabolism (Knechtle et al. 2008b), sleep and sleep deprivation (Knechtle et al. 2012b; Lahart et al. 2013), recovery (Neubauer et al. 2008), pain tolerance (Freund et al. 2013), association between anthropometry and performance (Knechtle et al. 2010), changes in body composition (Herbst et al. 2011; Knechtle et al. 2008a, b; Mueller et al. 2013; Schütz et al. 2013), and overuse injuries of the lower limbs (Freund et al. 2012) were not included.

\section{Conclusions}

This study showed that performance decreased linearly across days for Deca Iron ultra-triathletes (i.e. positive pacing) while performance remained unchanged across days for Triple Deca Iron ultra-triathletes (i.e. even pacing). To be successful in a Triple Deca Iron ultra-triathlon, a high number and a fast personal best time in ultratriathlons shorter than the Triple Deca Iron ultra-triathlon seem mandatory. We assume that ultra-triathletes successfully competing in longer races than a Deca Iron ultratriathlon such as a Triple Deca Iron ultra-triathlon need to gain experience in the long ultra-triathlon distances such as a Deca Iron ultra-triathlon.

Table 2 Number of finished ultra-triathlons with personal best time for Double Iron, Triple Iron and longer distances for finishers in the Triple Deca Iron ultra-triathlon

\begin{tabular}{|c|c|c|c|c|c|}
\hline Rank & $\begin{array}{c}\text { Number of finished } \\
\text { Triple Iron } \\
\text { ultra-triathlons }\end{array}$ & $\begin{array}{l}\text { Personal best } \\
\text { time Triple Iron } \\
\text { ultra-triathlon }\end{array}$ & $\begin{array}{c}\text { Number of finished } \\
\text { Double Iron } \\
\text { ultra-triathlons }\end{array}$ & $\begin{array}{l}\text { Personal best } \\
\text { time Double Iron } \\
\text { ultra-triathlon }\end{array}$ & $\begin{array}{l}\text { Other completed } \\
\text { ultra-triathlons }\end{array}$ \\
\hline 1 & - & - & 4 & $25: 47 \mathrm{~h}: \min$ & 1 Deca Iron in 268:12 h:min \\
\hline 2 & 1 & 40:14 h:min & 4 & 23:55 h:min & $\begin{array}{c}1 \text { Deca Iron in 222:17 h:min and } 1 \text { Double } \\
\text { Deca Iron in 481:54 h:min }\end{array}$ \\
\hline 3 & - & - & - & - & 1 Double Deca Iron in 497:56 h:min \\
\hline 4 & 1 & $36: 27$ h:min & 3 & 23:45 h:min & - \\
\hline 5 & - & - & - & - & - \\
\hline 6 & - & - & 8 & $28: 32 \mathrm{~h}: \min$ & 1 Deca Iron in 267:05 h:min \\
\hline 7 & 1 & 47:03 h:min & 1 & 29:05 h:min & - \\
\hline 8 & 2 & 47:58 h:min & 2 & 28:56 h:min & - \\
\hline
\end{tabular}




\section{Competing interests}

The authors declare that they have no competing interests.

\section{Authors' contributions}

BK collected all data and drafted the manuscript. TR helped in the interpretation of the data and helped drafting the manuscript. $R L$ and $C R$ performed the statistical analyses. All authors read and approved the final manuscript.

\section{Author details}

${ }^{1}$ Institute of Primary Care, University of Zurich, Zurich, Switzerland. ${ }^{2}$ Gesundheitszentrum St. Gallen, Vadianstrasse 26, 9001 St. Gallen, Switzerland. ${ }^{3}$ INSERM U1093, Faculty of Sport Sciences, University of Burgundy, Dijon, France.

Received: 30 January 2014 Accepted: 18 August 2014

Published: 24 August 2014

\section{References}

Abbiss CR, Laursen PB (2008) Describing and understanding pacing strategies during athletic competition. Sports Med 38:239-252

Dempster S, Britton R, Murray A, Costa RJS (2013) Case study: nutrition and hydration status during $4,254 \mathrm{~km}$ of running over 78 consecutive days. Int J Sport Nutr Exerc Metab 23:533-541

Freund W, Weber F, Billich C, Schuetz UH (2012) The foot in multistage ultra-marathon runners: experience in a cohort study of 22 participants of the Trans Europe Footrace Project with mobile MRI. BMJ Open 22:2(3)

Freund W, Weber F, Billich C, Birklein F, Breimhorst M, Schuetz UH (2013) Ultra-marathon runners are different: investigations into pain tolerance and personality traits of participants of the TransEurope FootRace 2009. Pain Pract 13:524-532

Herbst L, Knechtle B, Lopez CL, Andonie JL, Fraire OS, Kohler G, Rüst CA, Rosemann T (2011) Pacing strategy and change in body composition during a Deca Iron triathlon. Chin J Physiol 54:255-263

IUTA International ultra-triathlon association website., www.iutasport.com/archives/ results.php

Knechtle B, Salas Fraire O, Andonie JL, Kohler G (2008a) Effect of a multistage ultra-endurance triathlon on body composition: World Challenge Deca Iron Triathlon 2006. Br J Sports Med 42:121-125

Knechtle B, Knechtle P, Schück R, Andonie JL, Kohler G (2008b) Effects of a Deca Iron Triathlon on body composition: a case study. Int J Sports Med 29:343-351

Knechtle B, Wirth A, Baumann B, Knechtle P, Rosemann T, Oliver S (2010) Differential correlations between anthropometry, training volume, and performance in male and female Ironman triathletes. J Strength Cond Res 24:2785-2793

Knechtle B, Knechtle P, Lepers R (2011a) Participation and performance trends in ultra-triathlons from 1985 to 2009. Scand J Med Sci Sports 21:e82-e90

Knechtle B, Knechtle P, Rosemann T, Senn O (2011b) Personal best time, not anthropometry or training volume, is associated with total race time in a triple iron triathlon. J Strength Cond Res 25:1142-1150

Knechtle B, Morales NP, González ER, Gutierrez AA, Sevilla JN, Gómez RA, Robledo AR, Rodríguez AL, Fraire OS, Andonie JL, Lopez LC, Kohler G, Rosemann T (2012a) Effect of a multistage ultraendurance triathlon on aldosterone, vasopressin, extracellular water and urine electrolytes. Scott Med J 57:26-32

Knechtle B, Wirth A, Knechtle P, Rüst CA, Rosemann T, Lepers R (2012b) No improvement in race performance by naps in male ultra-endurance cyclists in a 600-km ultra-cycling race. Chin J Physiol 55:125-133

Knechtle B, Rüst CA, Rosemann T, Martin N (2014) 33 Ironman triathlons in 33 days-a case study. Springerplus 3:269

Lahart IM, Lane AM, Hulton A, Williams K, Godfrey R, Pedlar C, Wilson MG, Whyte GP (2013) Challenges in maintaining emotion regulation in a sleep and energy deprived state induced by the $4800 \mathrm{Km}$ ultra-endurance bicycle race; The Race Across AMerica (RAAM). J Sports Sci Med 12:481-488

Lepers R (2008) Analysis of Hawaii ironman performances in elite triathletes from 1981 to 2007. Med Sci Sports Exerc 40:1828-1834

Lepers R, Knechtle B, Knechtle P, Rosemann T (2011) Analysis of ultra-triathlon performances. Open Access J Sports Med 2:131-136

Mueller SM, Anliker E, Knechtle P, Knechtle B, Toigo M (2013) Changes in body composition in triathletes during an Ironman race. Eur J Appl Physiol $113: 2343-2352$
Neubauer O, König D, Wagner KH (2008) Recovery after an Ironman triathlon: sustained inflammatory responses and muscular stress. Eur J Appl Physiol 104:417-426

Reinboud W (2004) Linear models can't keep up with sport gender gap. Nature 432(7014):147

Schütz UH, Billich C, König K, Würslin C, Wiedelbach H, Brambs HJ, Machann J (2013) Characteristics, changes and influence of body composition during a $4486 \mathrm{~km}$ transcontinental ultramarathon: results from the Transeurope Footrace mobile whole body MRI-project. BMC Med 11:122

St Clair Gibson A, Lambert EV, Rauch LH, Tucker R, Baden DA, Foster C, Noakes TD (2006) The role of information processing between the brain and peripheral physiological systems in pacing and perception of effort. Sports Med 36:705-722

Zaryski C, Smith DJ (2005) Training principles and issues for ultra-endurance athletes. Curr Sports Med Rep 4:165-170

doi:10.1186/2193-1801-3-46

Cite this article as: Knechtle et al:: A comparison of performance of Deca Iron and Triple Deca Iron ultra-triathletes. SpringerPlus 2014 3:461.

\section{Submit your manuscript to a SpringerOpen ${ }^{\circ}$ journal and benefit from:}

- Convenient online submission

- Rigorous peer review

- Immediate publication on acceptance

- Open access: articles freely available online

- High visibility within the field

- Retaining the copyright to your article

Submit your next manuscript at $>$ springeropen.com 